\title{
Functionalization of Silica Surface with UV-Active Molecules by Multivalent Organosilicon Spacer
}

\author{
Olga Iliashevsky, Elina Rubinov, Yafa Yagen, Moshe Gottlieb* \\ Chemical Engineering Department, Ben Gurion University of the Negev, Beersheva, Israel \\ Email: *mosheg@bgu.ac.il
}

Received 23 April 2016; accepted 25 June 2016; published 28 June 2016

Copyright (C) 2016 by authors and Scientific Research Publishing Inc.

This work is licensed under the Creative Commons Attribution International License (CC BY). http://creativecommons.org/licenses/by/4.0/

c) (†) Open Access

\begin{abstract}
Immobilization of active molecules by attachment onto solid surfaces is a well-established practice in many processes and applications. Silica micro- and nano-particles are attractive candidates as support for active molecules as a result of a combination of desirable properties. The present study deals with grafting of a functional UV active molecule onto silica surfaces via hydrosilylation reactions using multivalent organosilicon spacers. Different types of organosilicon precursors based on dimethylsiloxysilanes containing multiple SiH groups were used as spacers between vinyl-modified silica surface and the benzotriazole UV-absorber (UVA). Firstly, the surface of silica was modified with vinyltrimetoxsisilane coupling agent. The UVA molecules were attached to the silica-vinyl by a two-step procedure involving hydrosilylation reaction. The successful grafting was confirmed by FTIR, TGA and UV-Vis characterization. More than fivefold increase in UVA loading was found for the MH30 multifunctional spacer, which contains approximately $75 \mathrm{SiH}$ groups per molecule, when compared to a four-functional spacer. Furthermore a branched, bulky six-functional spacer is preferred over a linear spacer of the same functionality. UV activity of functionalized silica was studied by UV-Vis spectroscopy, indicating that the immobilization of UVA onto silica surface has not altered its UV absorbance properties.
\end{abstract}

\section{Keywords}

Silica, Functionalized Silica, UV Absorbers, Hydrosilylation, Organosilicon

\section{Introduction}

Immobilization of active molecules by attachment onto solid surfaces in order to prevent their loss, facilitate

${ }^{*}$ Corresponding author.

How to cite this paper: Iliashevsky, O., Rubinov, E., Yagen, Y. and Gottlieb, M. (2016) Functionalization of Silica Surface with UV-Active Molecules by Multivalent Organosilicon Spacer. Open Journal of Inorganic Chemistry, 6, 163-174. 
their extraction and recycling, or prevent migration and volatilization, is a well-established practice in many processes and applications. Silica micro- and nano-particles are attractive candidates as support for active molecules as a result of a combination of desirable properties: 1) a large number of surface silanol groups available for a myriad of coupling reactions, 2) particles readily available in a variety of sizes and aggregation states from a large number of commercial suppliers, 3) silica inertness, and 4) favorable economics. As a result, functionalized silica has found many applications in the fields of catalysis [1], chromatography [2] [3], carriers of bioactive molecules [4] [5], fillers in polymer based products [6] [7], and dispersants [8] to name a few. Still, a number of desirable organic molecules are devoid of a reactive group suitable for covalent grafting onto the silica surface as in the case of organic UV absorbing molecules commonly used as light-stabilizers in polyolefins. Because of their relatively low molecular weight and thermodynamic incompatibility with the substrate, they are easy to volatilize during polymer processing or usage and they tend to migrate and bleed-out onto the article surface after relatively short times.

To overcome the inability to directly react the functional molecules with the surface silanol groups, the latter are reacted with organosilane coupling reagents that act as precursors for further immobilization [9] [10]. Nonetheless, typically due to steric hindrance during the reaction between the silane precursor and the functional molecule, this synthetic pathway leads to a relatively low loading of functional molecules. A possible way to improve the organic loading on the silica surface is by the utilization of multivalent precursors [11] [12].

The present study deals with grafting of a functional UV active molecule on silica particle surfaces via hydrosilylation reactions using multivalent organosilicon spacers. Different types of organosilicon precursors based on dimethylsiloxysilanes containing multiple $\mathrm{SiH}$ groups were used as spacers between vinyl-modified silica surface and the UV-absorber (UVA) molecules. 2-(2H-benzotriazol-2-yl)-4-methyl-6-(2-propenyl) phenol that belongs to the benzotriazole family, the most widely investigated and used group of UVA [13], was chosen as the UV active molecule due to its ability to undergo hydrosilylation reaction with $\mathrm{SiH}$ groups without the loss of UV-absorbing properties. Firstly, the surface of silica was modified with vinyltrimetoxysilane coupling agent (Scheme 1). The pre-activated silica-vinyl was fully characterized using complementary analytical methods.

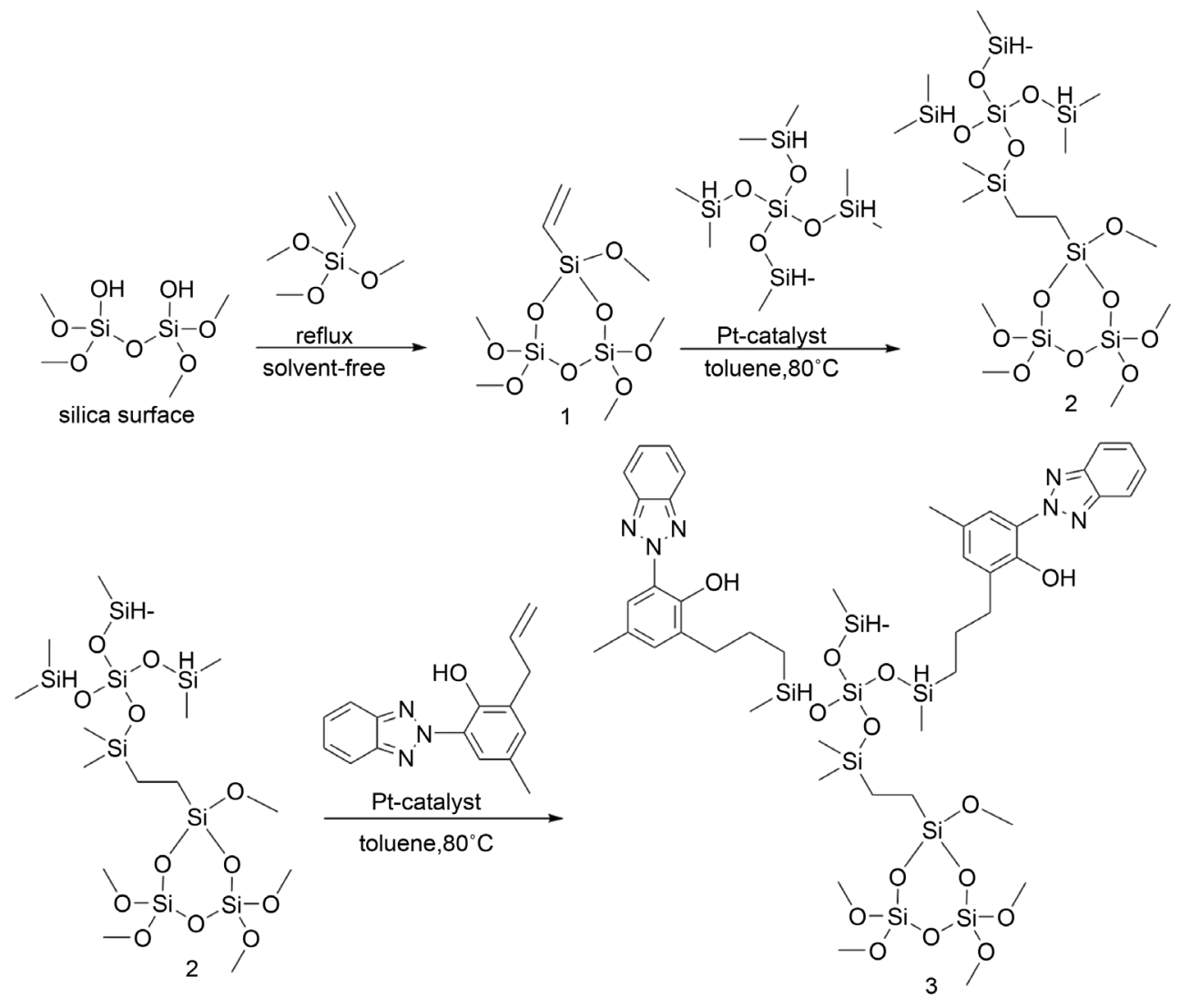

Scheme 1. Functionalization of vinyl modified silica with UVA through A4 spacer. 
The UVA molecules were attached to the silica-vinyl by a two-step procedure involving hydrosilylation reaction. The successful grafting was confirmed by FTIR, TGA and UV-Vis characterization.

\section{Experimental}

\subsection{Materials and Methods}

Fumed silica Cab-O-Sil ${ }^{\circledR}$ M5 with an average primary particle diameter of $14 \mathrm{~nm}$ and a specific surface area of $200 \mathrm{~m}^{2} \cdot \mathrm{g}^{-1}$, was obtained from Cabot Corporation (USA). The surface silanol group concentration of the Cab-O$\mathrm{Sil}^{\circledR}$ M5 material was determined by TGA analysis. The silica powder was pressed to pellets using a $\mathrm{KBr}$ pellet kit under pressure ( 2.5 ton) for 10 minutes. The pellets were pulverized and inserted into the TGA crucible. The weight loss of the silica powder sample over a temperature range of $298 \mathrm{~K}-1373 \mathrm{~K}$ was used to extract the silanol concentration following the method of Ek et al. [14]. The measurements yielded a value of $1.36 \mathrm{mmol}$ of silanol groups per gram of silica which corresponds in our case, to $4.1 \mathrm{OH}$ groups $\mathrm{nm}^{-2}$ not different from 4.6 $4.9 \mathrm{OH} \cdot \mathrm{nm}^{-2}$ [15] and $3.7-5.2 \mathrm{OH} \cdot \mathrm{nm}^{-2}$ [16], the values reported in the literature.

Polymethylhydrosiloxane Andisil ${ }^{\circledR}$ MH30 (MH30) was obtained from AB Specialty Silicones (IL, USA). The number average molecular weight of MH30 is $\mathrm{M}_{\mathrm{n}}=4414 \mathrm{gr} \cdot \mathrm{mol}^{-1}$, determined by GPC calibrated with PDMS standards (PSS, Germany). The number of $\mathrm{SiH}$ units per MH30 chain is 75 , as determined by ${ }^{1} \mathrm{H}-\mathrm{NMR}$ with dioxane as an internal standard and in close agreement with the independently determined $\mathrm{Mn}$ value.

VTMS-vinyltrimethoxysilane (97\%), A6-trimethylsilyl terminated poly (dimethylsiloxane-co-methylhydrosiloxane) (Mn $950 \mathrm{~g} \cdot \mathrm{mol}^{-1}, 6$ hydrosiloxane groups per molecule), Pt-catalyst-cis-dichlorobis (diethyl sulfide) platinum (II), and UVA-2-(2H-benzotriazol-2-yl)-4-methyl-6-(2-propenyl) phenol (99\%) were purchased from Sigma-Aldrich.

A4-Tetrakis(dimethylsiloxy)silane (97\%) was supplied by ABCR GmbH (Germany), n-hexane (AR) was purchased from Bio-Lab (Israel), and toluene (AR) was purchased from Gadot (Israel). All solvents and reagents were used without further purification.

A6y-3,3'-(1,2-Ethanediyl)-bis(3-dimethylsilyloxy)-1,1,5,5-tetramethyl trisiloxane was prepared in our labs according to the procedure described in [17].

\subsection{Instrumentation}

Thermogravimetric analysis (TGA) was performed using a Mettler-Toledo model TGA/SDTA851. Typically, approximately $10 \mathrm{mg}$ of the sample were heated in a standard $70 \mu \mathrm{L}$ TGA alumina crucible, from $298 \mathrm{~K}$ to 1273 $\mathrm{K}$, with a heating rate of $10 \mathrm{~K} \cdot \mathrm{min}^{-1}$ under nitrogen atmosphere. The FTIR spectra in $\mathrm{KBr}$ pellets were recorded on a Thermo Scientific, Nicolet ${ }^{\mathrm{TM}}$ iS $^{\mathrm{TM}} 10$ FTIR spectrometer. Nuclear Magnetic Resonance (NMR) spectra were acquired on a Bruker Avance II instrument, $500 \mathrm{MHz}$, CP-MAS $4 \mathrm{~mm}$. X-ray photoelectron spectroscopy (XPS) was performed using a Kratos Analytical Ltd. (Kratos Axis Ultra) spectrometer. UV-Vis spectra were obtained using PerkinElmer, Lambda ${ }^{\mathrm{TM}} 35$ spectrophotometer.

\subsection{Synthesis}

\subsubsection{Modification of Silica Surface with Vinyl Groups}

Modification of silica surface with vinyltrimethoxysilane (VTMS) was carried out following a modified literature procedure [18] as follows: 10 gr of the pure silica and 40 gr of VTMS were mixed in a 3-necked flask fitted with a reflux condenser and $\mathrm{CaCl}_{2}$ trap. The mixture was heated to the boiling point of VTMS (b.p. $396 \mathrm{~K}$ ) using an oil bath $(383 \mathrm{~K})$, and was continuously stirred with a mechanical stirrer at $225 \mathrm{rpm}$ for 11 hours. After cooling to room temperature, the silica was washed three times with hexane $(3 \times 250 \mathrm{ml})$ in a filter funnel with sinter glass (P4) and was dried in a petri dish for half an hour in an oven at $438 \mathrm{~K}$ to obtain $9.56 \mathrm{gr}$ of silica-vinyl (1, Scheme 1).

\subsubsection{Functionalization of Silica-Vinyl with UVA}

1) General procedure: silica-spacer

The following procedure was used in all the reactions described below: the spacer molecule was added to a suspension of silica-vinyl in toluene, followed by the addition of the Pt catalyst solution ( $2 \%$ in toluene). The 
resultant mixture was vigorously stirred for 5 hours at $353 \mathrm{~K}$. After cooling to room temperature, the product was washed three times with toluene and four times with hexane on a filter funnel equipped with sinter glass (P4). The product was placed in a petri dish and dried for one hour in an oven at $343 \mathrm{~K}$.

The silica-spacer obtained above was subsequently suspended in toluene. UVA was added to the suspension, followed by the addition of the Pt catalyst solution (2\% in toluene). The resultant mixture was stirred (225 rpm) for 8 hours at $353 \mathrm{~K}$. After cooling to room temperature, the product was washed four times with toluene and three times with hexane on a filter funnel with sinter glass (P4). UV absorbance of the last portion of hexane was measured (250 - $450 \mathrm{~nm}$ ) to ensure that all remaining UVA was washed away. The product was placed in a petri dish and dried for one hour in an oven at $343 \mathrm{~K}$.

2) Preparation of silica-A4

The following reagents and conditions were used for the synthesis of silica-A4: $10.00 \mathrm{gr}$ of silica-vinyl, $4.70 \mathrm{gr}$ of A4, 54 gr of toluene, $425 \mu \mathrm{l} \mathrm{Pt} \mathrm{catalyst} \mathrm{solution.} \mathrm{After} \mathrm{drying} 9.95 \mathrm{gr}$ of silica-A4 were obtained (2, Scheme 1).

Preparation of silica-A4-UVA

The following reagents and conditions were used for the synthesis of silica-A4-UVA: 9.45 gr of silica-A4, $5.33 \mathrm{gr}$ of UVA, $60 \mathrm{gr}$ of toluene, $445 \mu \mathrm{l} \mathrm{Pt}$ catalyst solution. The suspension was stirred (225 rpm) for 16 hours at $80^{\circ} \mathrm{C}$. After drying 9.29 gr of silica-A4-UVA were obtained (3, Scheme 1 ).

3) Preparation of silica-A6

The following reagents and conditions were used for the synthesis of silica-A6: 1.00 gr of silica-vinyl, $1.32 \mathrm{gr}$ of A6, 5.22 gr of toluene, $50 \mu \mathrm{l} \mathrm{Pt}$ catalyst solution. After drying $0.982 \mathrm{gr}$ of silica-A6 were obtained.

Preparation of silica-A6-UVA

The following reagents and conditions were used for the synthesis of silica-A6-UVA: $750.8 \mathrm{mg}$ of silica-A6, $424.8 \mathrm{mg}$ of UVA, $4.78 \mathrm{gr}$ of toluene, $36 \mu \mathrm{lt}$ catalyst solution. After drying $0.625 \mathrm{gr}$ of silica-A6-UVA were obtained.

4) Preparation of silica-A6y

The following reagents and conditions were used for the synthesis of silica-A6y: 1.00 gr of silica-vinyl, 780 mg of A6y, 3.6 gr of toluene, $36 \mu \mathrm{l}$ Pt catalyst solution. After drying 0.984 gr of silica-A6y were obtained.

Preparation of silica-A6y-UVA

The following reagents and conditions were used for the synthesis of silica-A6y-UVA: $627.8 \mathrm{mg}$ of silica-A6y, $354.8 \mathrm{mg}$ of UVA, 2.26 gr of toluene, $28 \mu \mathrm{l}$ Pt catalyst solution. After drying 0.518 gr of silica-A6yUVA were obtained.

5) Preparation of silica-MH30

The following reagents and conditions were used for the synthesis of silica-MH30: 1.00 gr of silica-vinyl (pre-dried for $1 \mathrm{~h}$ at $433 \mathrm{~K}$ ), 3.29 gr of MH30, 2.6 gr of toluene, and $45 \mu \mathrm{lt}$ catalyst solution. The resultant suspension was stirred for 8 hours at $323 \mathrm{~K}$ with a magnetic stirrer. The product was placed in a petri dish and dried in an oven at $318 \mathrm{~K}$ for one hour, to obtain $1.2 \mathrm{gr}$ of silica-MH30.

Preparation of silica-MH30-UVA

The following reagents and conditions were used for the synthesis of silica-MH30-UVA: 0.5 gr of silicaMH30, 1.5 gr of UVA, 3 gr of toluene, $80 \mu \mathrm{l}$ of Pt catalyst solution. After drying $0.45 \mathrm{gr}$ of silica-MH30-UVA were obtained.

\section{Results and Discussion}

\subsection{Characterization of Vinyl-Modified Silica}

Solid-state ${ }^{13} \mathrm{C}$ NMR was used to characterize the chemistry of the silica surface. The NMR spectrum of vinylmodified silica exhibits clearly the methoxy signal at ca. 48 ppm and two signals at ca. 134 - 128 ppm which correspond to the carbons of the vinyl groups on the silica surface as shown in Figure 1.

The FTIR spectra for the silica before and after reaction with VTMS are presented in Figure 2. The characteristic peaks of the unmodified silica are: a large band at about $3433 \mathrm{~cm}^{-1}$, attributed to the O-H stretching frequency of silanol groups and to adsorbed water; a sharp band at $1631 \mathrm{~cm}^{-1}$ assigned to the bending vibrations of molecular water; a broad strong peak at $1254-1026 \mathrm{~cm}^{-1}$, assigned to the Si-O-Si asymmetric bond stretching vibrations. This peak has a distinct "shoulder" at $\sim 980 \mathrm{~cm}^{-1}$, which corresponds to the asymmetric vibration of $\mathrm{Si}-\mathrm{OH}$. A band at $806 \mathrm{~cm}^{-1}$ is assigned to a network Si-O-Si symmetric bond stretching vibration; and the band at $460 \mathrm{~cm}^{-1}$ is associated with a network Si-O-Si bond bending vibration. New weak peaks at $3060 \mathrm{~cm}^{-1}$ and $3020 \mathrm{~cm}^{-1}$ are observed in the spectra for the vinyl-modified silica. These are attributed to C-H bands of the 


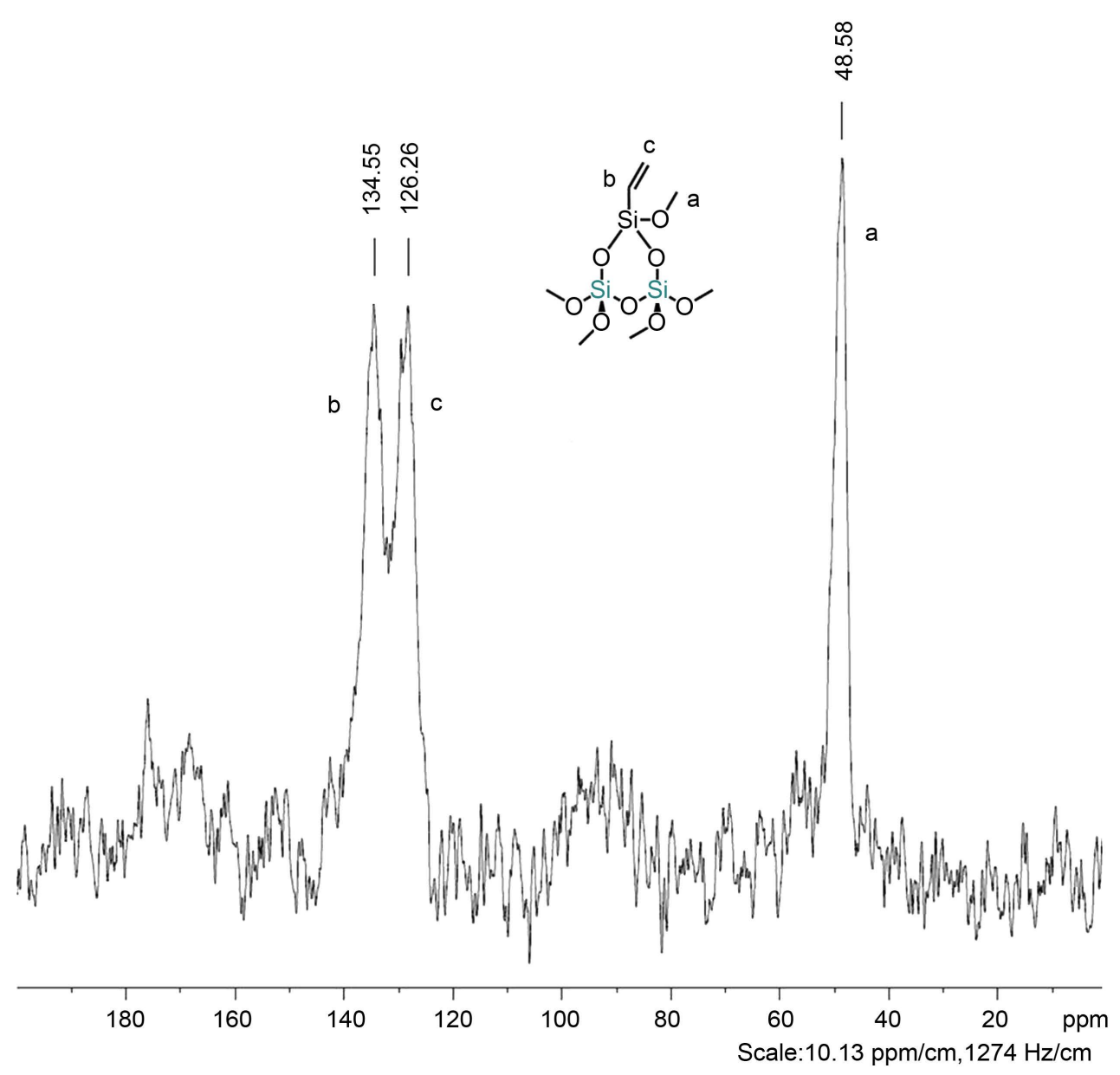

Figure $1 .{ }^{13} \mathrm{C}$ CPMAS NMR of vinyl-modified silica. Assignments are as follows: 48.58 ppm-methoxy group; 128.26 and 134.55-vinyl group (distal and proximal carbon atoms respectively).

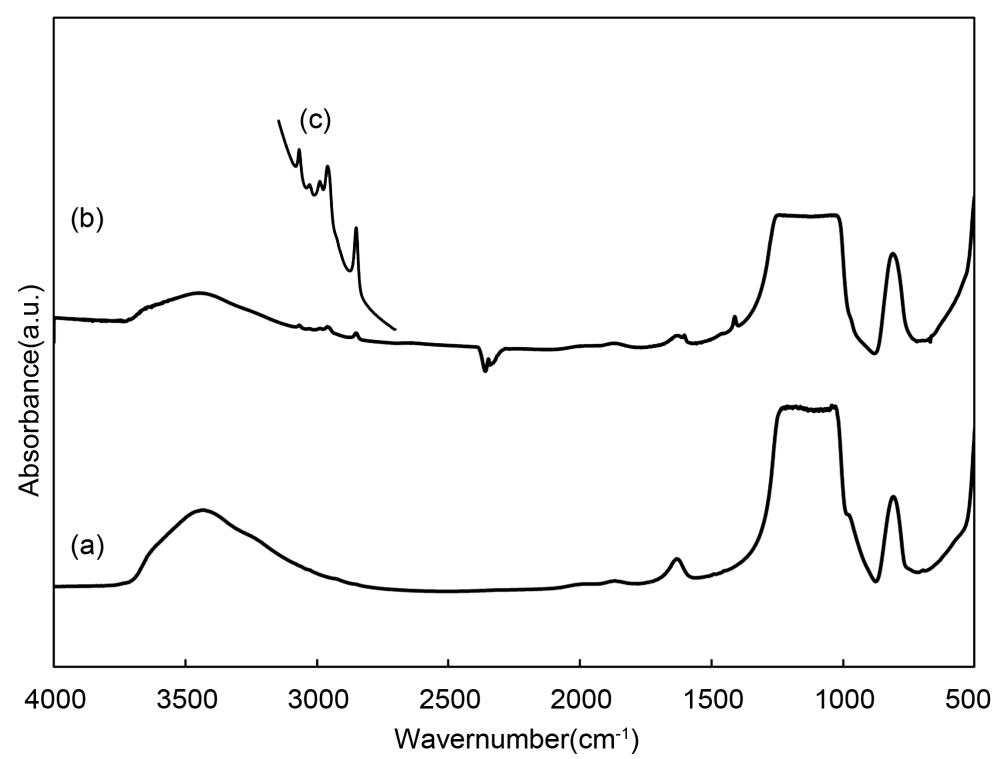

Figure 2. FTIR spectra for (a) pristine Cab-O-Sil M5, (b) silica-vinyl, vinyl modified silica. Magnification of spectrum (b) between $2700 \mathrm{~cm}^{-1}$ and $3150 \mathrm{~cm}^{-1}$ is shown as curve (c). Relevant assignments are discussed in the text. 
$\mathrm{Si}-\mathrm{CH}=\mathrm{CH}_{2}$ group. The peak at $1600 \mathrm{~cm}^{-1}$ is typical band of $\mathrm{C}=\mathrm{C}$ vibrations and the peak appearing at 1410 $\mathrm{cm}^{-1}$ is assigned to C-H deformation vibrations of the $=\mathrm{CH}_{2}$ group. The peaks at $2986 \mathrm{~cm}^{-1}, 2958 \mathrm{~cm}^{-1}$ and 2847 $\mathrm{cm}^{-1}$ are attributed to $\mathrm{C}-\mathrm{H}$ bands of the unreacted methoxy groups of the VTMS (the characteristic $\mathrm{Si}-\mathrm{OCH}_{3}$ band, expected to be found around $1000-1100 \mathrm{~cm}^{-1}$, is masked by the strong Si-O-Si absorption). Lastly, it should be pointed out that in the modified silica, the broad band at $3433 \mathrm{~cm}^{-1}$ is significantly decreased in comparison to that of the original sample. This decrease, along with the disappearance of the "shoulder" at 980 $\mathrm{cm}^{-1}$, clearly indicate that surface silanol groups have been consumed in the chemical reaction, i.e., vinyltrimethoxysilane was covalently anchored to the silica surface by reaction with the surface silanol groups.

The successful attachment of vinyltrimethoxysilane to the surface of the silica particles is also demonstrated by the XPS analysis. The modification of the silica surface is expected to lead to a significant decreased oxygen/ carbon and silicon/carbon ratios measured on the silica surface. This is indeed shown by the results set forth in Table 1.

The chemical composition of the vinyl-modified silica was further studied using high resolution C1s spectrum. Two main bands corresponding to the following bonds: $\mathrm{C}-\mathrm{H}(285 \mathrm{eV})$ and $\mathrm{O}-\mathrm{CH}_{3}(287 \mathrm{eV})$ were obtained (Table 2). Based on the deconvoluted band areas, the ratio between the $\mathrm{C}=\mathrm{C}$ and $\mathrm{C}-\mathrm{O}$ was found to be approximately 2:1. The ratio of the two carbon bands reflects the proportion of the vinyl to methoxy groups. Therefore, we can estimate that on average there is one vinyl group to one methoxy group per each grafted VTMS molecule on the silica surface. Hence we conclude that on the average two out of the three methoxy groups in a VTMS molecule have reacted and one is left unreacted (as also indicated by the FTIR spectra above).

The quantitative determination of the extent of VTMS incorporation onto the silica surface was achieved by thermogravimetric analysis. The dehydroxylation [15] [16] which may occur in this temperature range, is neglected since most of the silanol groups are assumed to have reacted during the functionalization reaction. NMR and FTIR results clearly indicate that not all the alkoxy groups are hydrolyzed, therefore it should be taken into account in the TGA analysis employed for the determination of the amount of surface vinyl groups. In combination with the XPS results discussed above a reliable account for the organic content decomposed during TGA analysis may be obtained. The value of $M=58 \mathrm{~g} \cdot \mathrm{mol}^{-1}$ is used for the molecular weight of the decomposed organic molecule based on one vinyl group and one methoxy group per VTMS molecule. The following equation was used to derive the concentration of vinyl groups on the silica surface in terms of $C_{V i}$, mmol vinyl groups/g-sample (silica + VTMS):

$$
C_{V i}\left(\mathrm{mmol} / \mathrm{g}_{\text {sample }}\right)=10^{3} f_{V i} / M
$$

where, $f_{v i}$ is the weight fraction of sample decomposed in the temperature range from 423 - $1273 \mathrm{~K}$. The TGA thermogram for the vinyl modified silica is presented in Figure 3. A vinyl surface concentration of $0.68 \mathrm{mmol} \cdot \mathrm{g}^{-1}$ was obtained from Equation (1). This value should be compared to the concentration of surface silanols determined above $\left(1.36 \mathrm{mmol} \cdot \mathrm{g}^{-1}\right)$. It indicates that approximately two silanol groups have been consumed for every VTMS molecule attached to the surface, in agreement with previous discussion.

\subsection{Characterization of Silica-Spacer-UVA}

TGA analysis was used to calculate the amount of UVA molecules attached to the silica surface at the end of

Table 1. Atomic concentrations, $\mathrm{O} / \mathrm{C}$ and $\mathrm{Si} / \mathrm{C}$ ratios obtained by XPS.

\begin{tabular}{ccccccc}
\hline Sample & O1s (\%) & N1s (\%) & C1s (\%) & Si2p (\%) & O/C & Si/C \\
\hline Pristine silica & 70.0 & 0 & 1.5 & 28.6 & 47.9 & 19.6 \\
Silica-vinyl & 63.2 & 0 & 9.6 & 27.2 & 6.6 & 2.8 \\
\hline
\end{tabular}

Table 2. Deconvoluted band areas of the vinyl-modified silica obtained from high resolution C1s XPS.

\begin{tabular}{ccc}
\hline Atom & Position $(\mathrm{eV})$ & Area (\%) \\
\hline C1s (C-H) & 285 & 66 \\
C1s (C-O) & 287 & 34 \\
\hline
\end{tabular}


sequence of reactions described above. The difference in weight loss between the silica-spacer particles and UVA functionalized silica particles was taken as the content of UVA that has been incorporated into the silica. Thus, the concentration of UVA molecules per gram of the functionalized silica, $C_{\mathrm{UVA}}\left(\mathrm{mmol} \cdot \mathrm{g}^{-1}\right)$, was calculated by the following equation.

$$
C_{\mathrm{UVA}}\left(\mathrm{mmol}-\mathrm{UVA} / \mathrm{g}_{\text {sample }}\right)=10^{3}\left(f_{\mathrm{UVA}}-f_{\text {spacer }}\right) /\left[\left(1-f_{\text {spacer }}\right) M_{\mathrm{UVA}}\right]
$$

where $f_{\text {spacer }}$ and $f_{\mathrm{UVA}}$ are the weight fractions of decomposed material in the temperature range $373-1273 \mathrm{~K}$ for a sample of silica-spacer, and a sample of silica-spacer-UVA respectively, and $M_{\mathrm{UVA}}$ is the molecular weight of UVA (265.31 $\left.\mathrm{g} \cdot \mathrm{mol}^{-1}\right)$. Results are summarized in Table 3.

The results presented in Table 3 indicate that relative to A4 there is more than fivefold increase in UVA loading for the MH30 spacer, which contains a considerably larger number of SiH groups. A6 and A6y also showed increase in the UVA concentration relative to A4. The "bulky" A6y spacer are preferred over the "linear" A6 spacer. The increase in UVA loading from A4 to A6y is roughly proportional to the increase in available $\mathrm{SiH}$ groups for UVA attachment ( 3 in the former, 5 in the latter).

It should be noted that UVA grafting improves the thermal stability of MH30 modified silica. Figure 4 represents TGA curves silica-MH30 and silica-MH-30-UVA. These curves indicate that the onset of decomposition of the organic components in silica-MH30 is at about $398 \mathrm{~K}$, while the decomposition in UVA functionalized silica occurs at a considerably higher temperature of about $598 \mathrm{~K}$. Thermograms for additional spacers are provided in the Appendix (cf. Figures A1-A3).

UVA functionalized silica was also examined by FTIR spectroscopy. Figure 5 shows spectra of UVA, silica-MH30 and silica-MH30-UVA. When comparing the spectra of silica-MH30 (Figure 5(b)) and silica-MH30-UVA (Figure 5(c)) new peaks are observed for the latter at $745 \mathrm{~cm}^{-1}$ and in the region from 1500 to

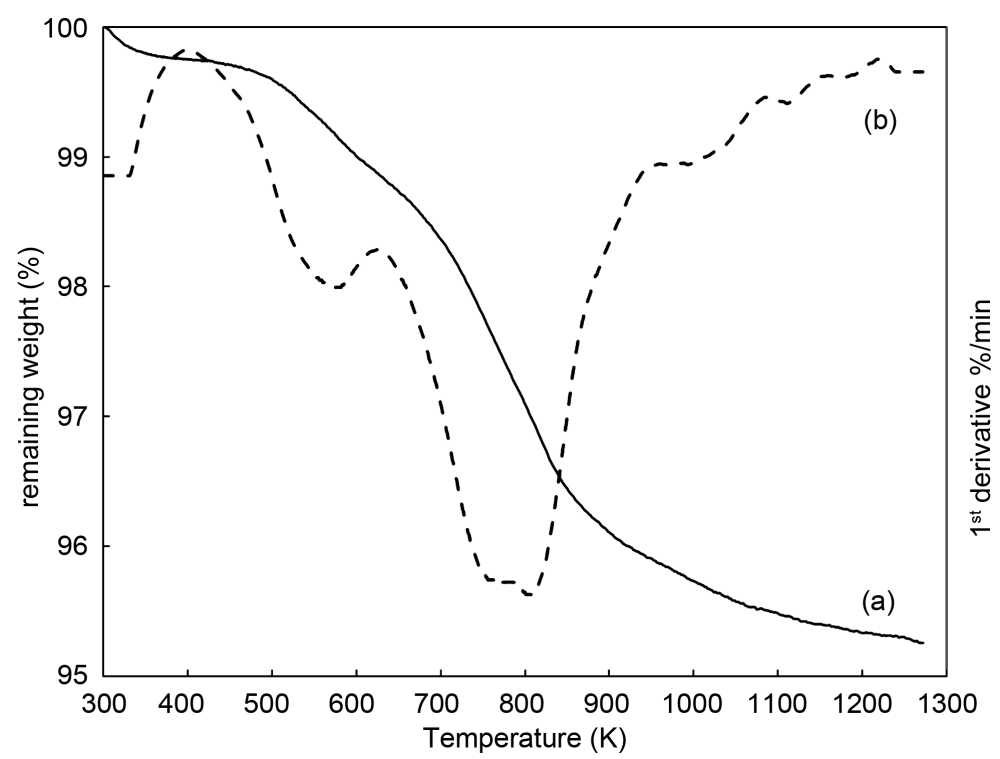

Figure 3. TGA diagram of vinyl-modified silica. (a) Percent of remaining sample weight, (b) $1^{\text {st }}$ derivative.

Table 3. Measured amount of UVA molecules attached to the silica for the different spacers used in this work ${ }^{\dagger}$.

\begin{tabular}{cccc}
\hline Sample & $f_{\text {spacer }}$ & $f_{\text {UVA }}$ & $C_{\text {UVA }}$ \\
\hline sillica-A4-UVA & 0.05 & 0.11 & 0.26 \\
silica-A6-UVA & 0.06 & 0.14 & 0.31 \\
silica-A6y-UVA & 0.06 & 0.19 & 0.51 \\
silica-MH30-UVA & 0.09 & 0.41 & 1.36 \\
\hline
\end{tabular}

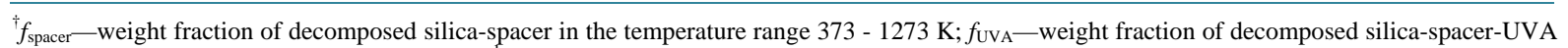
in the same temperature range; $C_{U V A}\left(\mathrm{mmol} \cdot \mathrm{g}^{-1}\right)$ concentration of UVA molecules per gram of functionalized silica. 


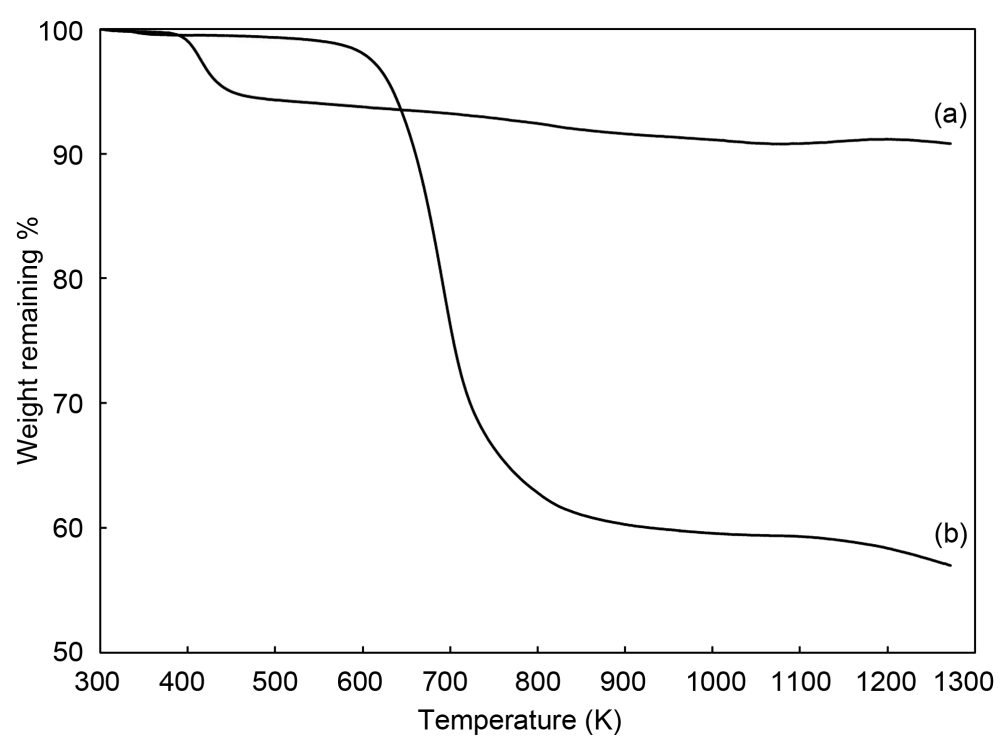

Figure 4. TGA thermograms for silica-MH30 (a), silica-MH30-UVA (b).

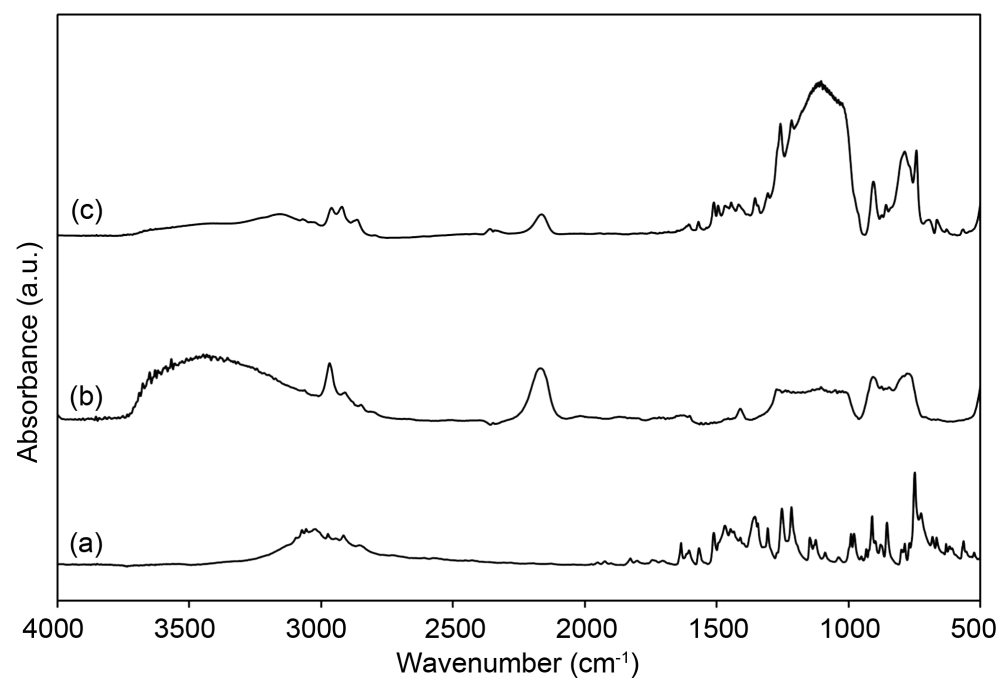

Figure 5. FTIR spectra of UVA (a), silica-MH30 (b) and silica-MH30-UVA (c).

$1200 \mathrm{~cm}^{-1}$. These peaks are characteristic to the UVA molecule (Figure 5(a)), indicating successful functionalization of the silica surface. The presence of the characteristic Si-H band at $2160 \mathrm{~cm}^{-1}$ is also detected in the spectrum of silica-MH30-UVA (Figure 5(c)) indicating that not all the SiH groups have been consumed by the hydrosilylation reaction. Spectra for all other materials prepared here are presented in Figures A4-A6 in the Appendix section.

Upon successful covalent functionalization of silica with UVA, its UV transmission properties were examined to ascertain its UV absorbing properties in typical applications. For this propose, silica-MH30-UVA was compounded into a polyethylene matrix to prepare $50 \mu \mathrm{m}$ thick, three-layered film with the additive incorporated into the $7 \mu \mathrm{m}$ thick core layer. The composition of the core layer consists of 93\% LLDPE and 7\% additive (by weight). The UV-VIS spectrum of the film is similar to that of the UVA molecule, with the characteristic absorption bands at $\lambda \sim 345 \mathrm{~nm}$ and $304 \mathrm{~nm}$, although some broadening of the bands is observed for the film (Figure 6).

\section{Conclusion}

UV active molecule has been successfully attached onto vinyl-modified silica surface using different types of 


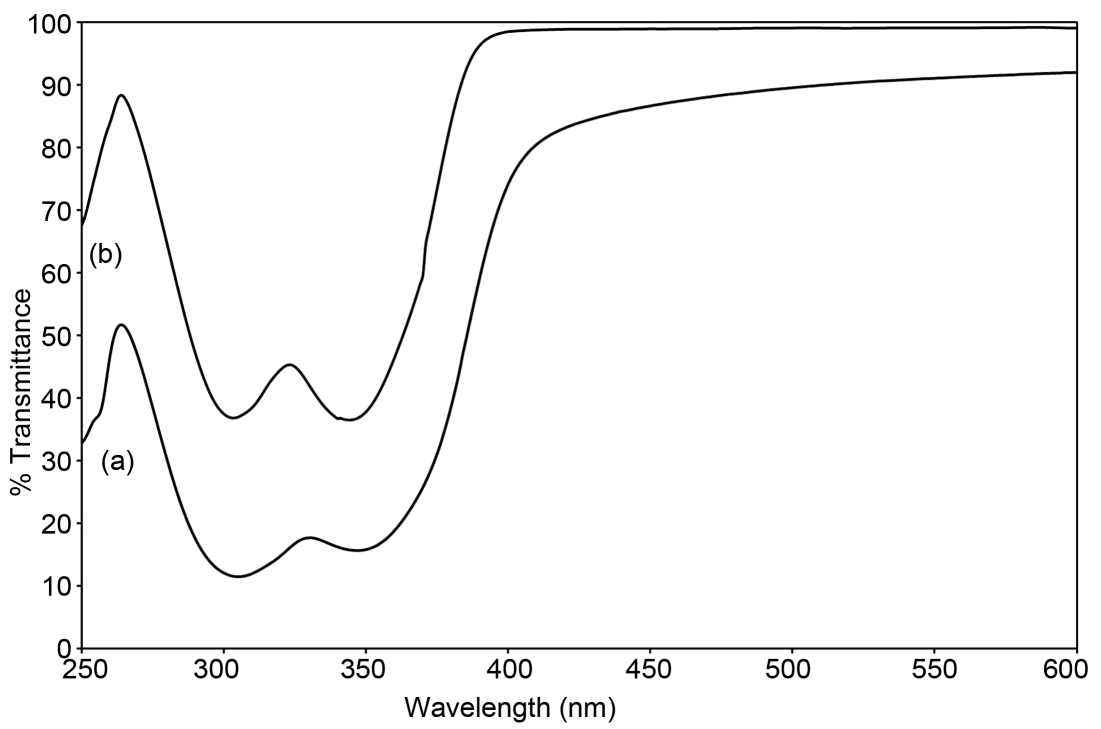

Figure 6. (a) UV-VIS spectra of $50 \mu \mathrm{m}$ multilayered LLDPE film with Sil-MH30UVA additive in the core layer; (b) 11.5 ppm hexane solution of UVA.

multivalent organosilicon precursors, which were used as a spacer between vinyl-modified silica surface and the UV-absorber. The new materials were characterized by TGA and FTIR. More than fivefold increase in UVA loading was found for the MH30 spacer, which contains a large number of SiH groups, when compared to a four-functional spacer. Six-functional spacers also showed increase in the UVA concentration compared to the four-functional spacer. Furthermore a branched, bulky six-functional spacer is preferred over a linear spacer of the same functionality. UV activity of functionalized silica was studied by UV-VIS spectroscopy, indicating that the immobilization of UVA onto silica surface has not altered its UV absorbance properties.

\section{Acknowledgements}

Financial support was provided by the CSO of the Israel Ministry of Economy and Industry in the framework of P^3 MAGNET program. The help provided by Ilia Mishsky and Anastasia Volison in some of the experimental aspects of this work are gratefully acknowledged.

\section{References}

[1] Price, P.M., Clark, J.H. and Macquarrie, D.J. (2000) Modified Silicas for Clean Technology. Journal of the Chemical Society, Dalton Transactions, 2, 101-110. http://dx.doi.org/10.1039/a905457j

[2] Scott, R.P.W. (1993) Silica Gel and Bonded Phases: Their Production, Properties and Use in LC. John Wiley \& Sons Ltd., Chichester.

[3] Jost, W. and Hauck, H.E. (1987) The Use of Modified Silica Gels in TLC and HPTLC. Advances in Chromatography, 27, 129-165.

[4] Barbé, C.., Bartlett, J., Kong, L. Finnie, K., Lin, H.Q., Larkin, M., Calleja, S., Bush, A. and Calleja, G. (2004) Silica Particles: A Novel Drug-Delivery System. Advanced Materials, 16, 1959-1966. http://dx.doi.org/10.1002/adma.200400771

[5] Zhao, Y., Trewyn, B.G., Slowing, I.I. and Lin, V.S.-Y. (2009) Mesoporous Silica Nanoparticle-Based Double Drug Delivery System for Glucose-Responsive Controlled Release of Insulin and Cyclic AMP. Journal of the American Chemical Society, 131, 8398-8400. http://dx.doi.org/10.1021/ja901831u

[6] Mark, J.E. (2007) Physical Properties of Polymers Handbook. 2nd Edition, Springer Science, New York. http://dx.doi.org/10.1007/978-0-387-69002-5

[7] ten Brinke, J.W., Debnath, S.C., Reuvekamp, L.A.E.M. and Noordermeer, J.W.M. (2003) Mechanistic Aspects of the Role of Coupling Agents in Silica-Rubber Composites. Composites Science and Technology, 63, 1165-1174. http://dx.doi.org/10.1016/S0266-3538(03)00077-0

[8] Greenwood, P. (2010) Modified Silica Sols: Titania Dispersants and Co-Binders for Silicate Paints. Pigment \& Resin 
Technology, 6, 315-321. http://dx.doi.org/10.1108/03699421011085803

[9] Plueddemann, E.P. (1991) Silane Coupling Agents. 2nd Edition, Plenum, New York. http://dx.doi.org/10.1007/978-1-4899-2070-6

[10] DeschIer, U., Kleinschmit, P. and Panster, P. (1986) 3-Chloropropyltrialkoxy Silanes-Key Intermediates for the Commercial Production of Organofunctionalized Silanes and Polysiloxanes. Angewandte Chemie International Edition, 25, 236-252. http://dx.doi.org/10.1002/anie.198602361

[11] Iliashevsky, O., Amir, L., Glaser, R., Marks, R.S. and Lemcoff, N.G. (2009) Synthesis, Characterization and Protein Binding Properties of Supported Dendrons. Journal of Materials Chemistry, 19, 6616-6622. http://dx.doi.org/10.1039/b908014g

[12] Schüll, C. and Frey, H. (2013) Grafting of Hyperbranched Polymers: From Unusual Complex Polymer Topologies to Multivalent Surface Functionalization. Polymer, 54, 5443-5455. http://dx.doi.org/10.1016/j.polymer.2013.07.065

[13] Crawford, J.C. (1999) 2(2-Hydroxyphenyl)2H-benzotriazole Ultraviolet Stabilizers. Progress in Polymer Science, 24, 7-43. http://dx.doi.org/10.1016/S0079-6700(98)00012-4

[14] Ek, S., Root, A., Peussa, M. and Niinistö, L. (2001) Determination of the Hydroxyl Group Content in Silica by Thermogravimetry and a Comparison with 1H MAS NMR Results. Thermochimica Acta, 379, 201-212. http://dx.doi.org/10.1016/S0040-6031(01)00618-9

[15] Zhuravlev, L.T. (2000) The Surface Chemistry of Amorphous Silica. Zhuravlev Model. Colloids and Surfaces A: Physicochemical and Engineering Aspects, 173, 1-38. http://dx.doi.org/10.1016/S0927-7757(00)00556-2

[16] Rozanska, X., Delbecq, F. and Sautet, P. (2010) Reconstruction and Stability of $\beta$-Cristobalite 001, 101, and 111 Surfaces during Dihydroxylation. Physical Chemistry Chemical Physics, 12, 14930-14940. http://dx.doi.org/10.1039/c0cp00287a

[17] Herzig, C., Deubzer, B., Bloechl, M. and Banfic, R. (1997) Crosslinkable Compositions. US Patent Number 5691435.

[18] Su, H.L., Hsu, J.M., and Pan, J.P. and Chern, C.S. (2007) Silica Nanoparticles Modified with Vinyltriethoxysilane and Their Copolymerization with N,N'-bismaleimide-4,4'-diphenylmethane. Journal of Applied Polymer Science, 103, 3600-3608. http://dx.doi.org/10.1002/app.25313 


\section{Appendix}

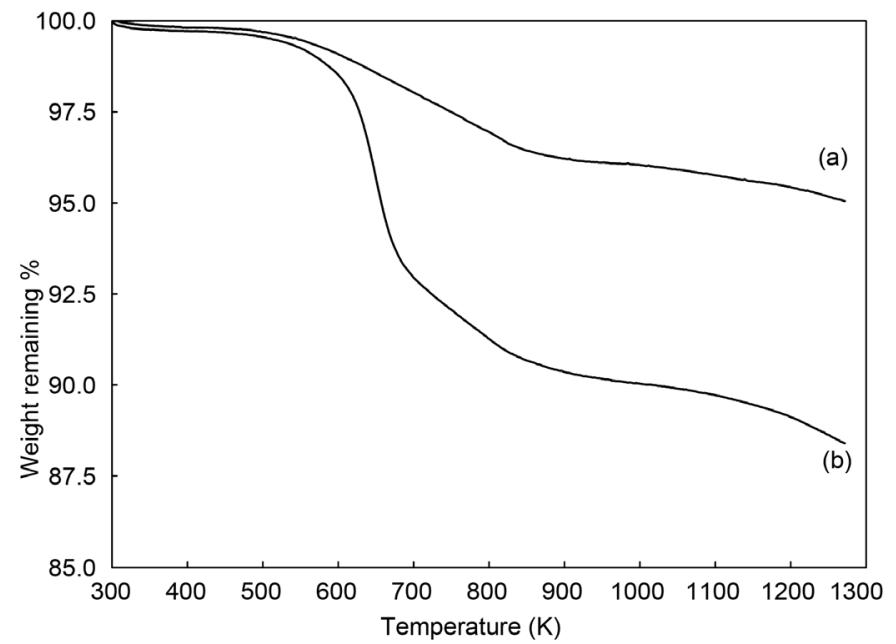

Figure A1. TGA thermograms for silica-A4 (a), silica-A4-UVA (b).

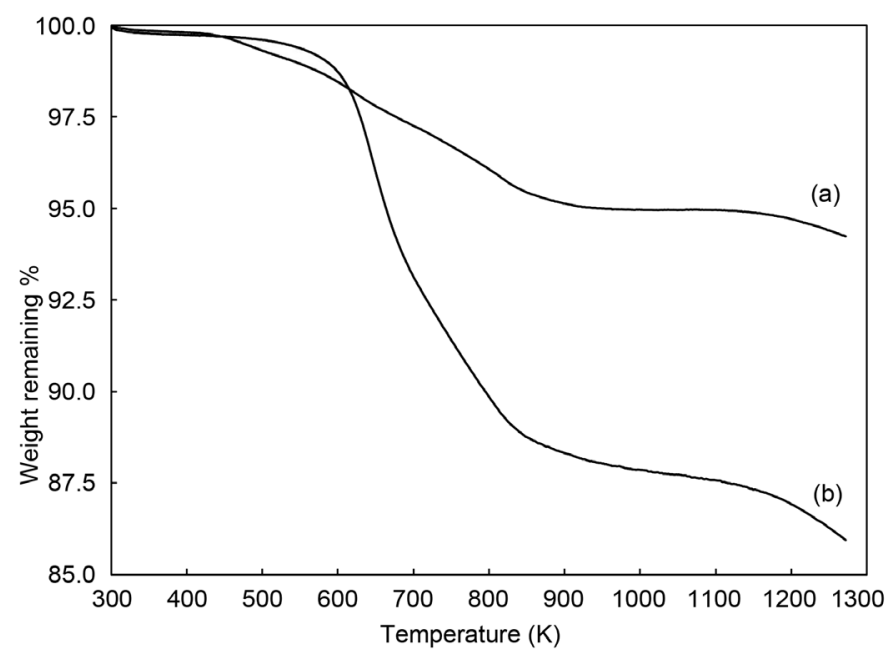

Figure A2. TGA thermograms for silica-A6 (a), silica-A6-UVA (b).

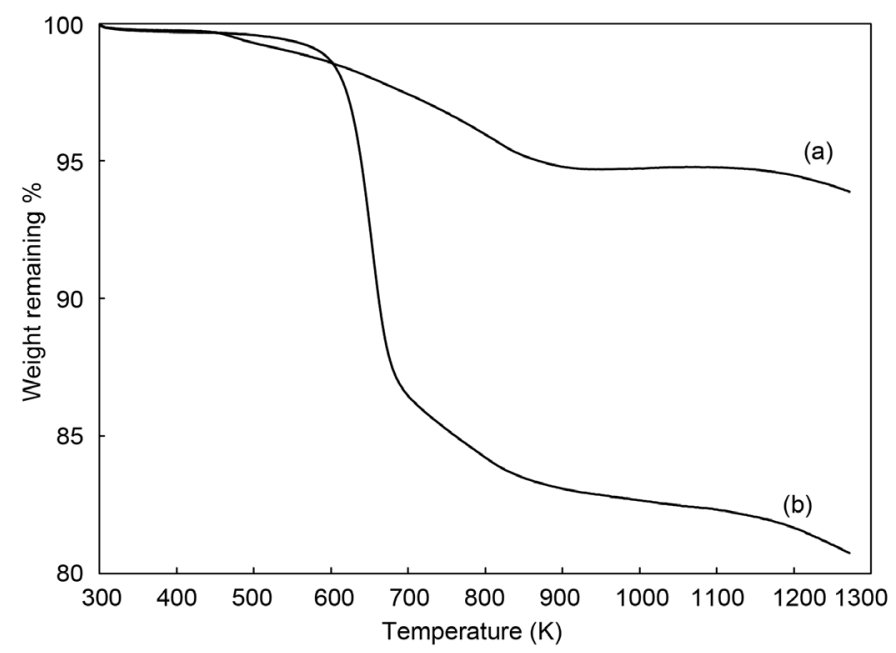

Figure A3. TGA thermograms for silica-A6y (a), silica-A6y-UVA (b). 


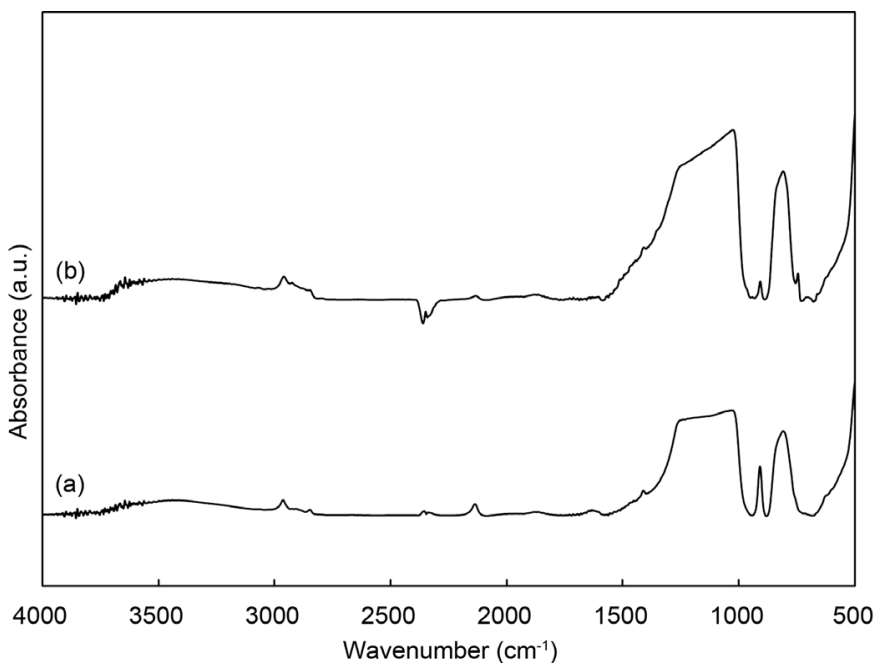

Figure A4. FTIR spectra for silica-A4 (a), silica-A4-UVA (b).

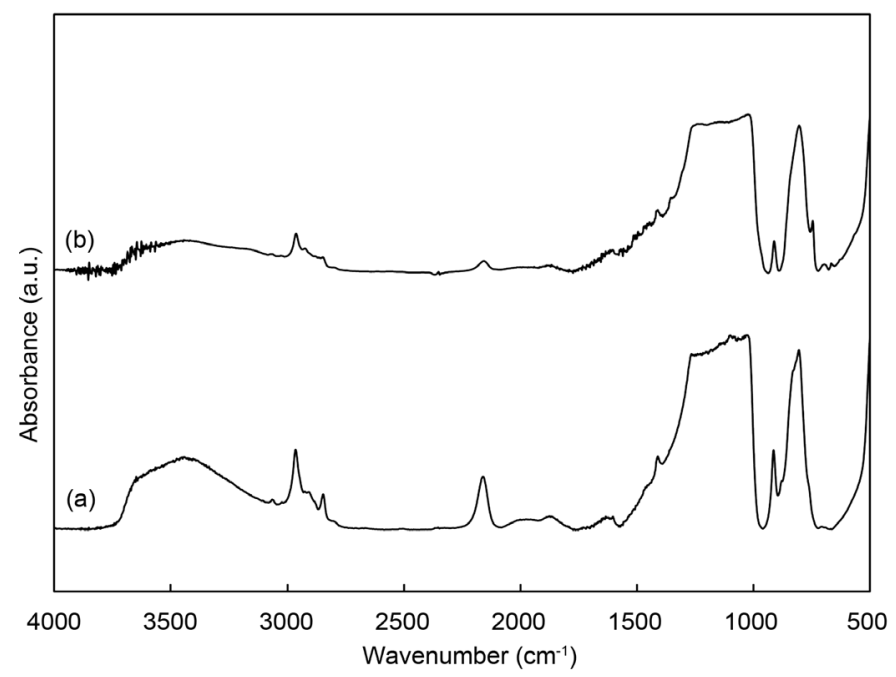

Figure A5. FTIR spectra for silica-A6 (a), silica-A6-UVA (b).

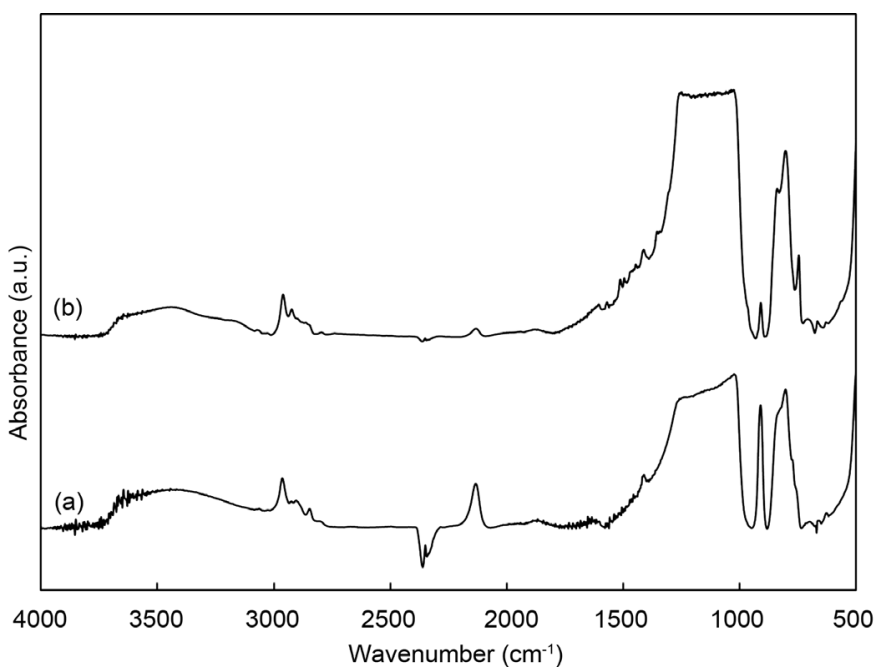

Figure A6. FTIR spectra for silica-A6y (a), silica-A6y-UVA (b). 


\section{Submit or recommend next manuscript to SCIRP and we will provide best service for you:}

Accepting pre-submission inquiries through Email, Facebook, Linkedin, Twitter, etc A wide selection of journals (inclusive of 9 subjects, more than 200 journals)

Providing a 24-hour high-quality service

User-friendly online submission system

Fair and swift peer-review system

Efficient typesetting and proofreading procedure

Display of the result of downloads and visits, as well as the number of cited articles

Maximum dissemination of your research work

Submit your manuscript at: http://papersubmission.scirp.org/ 